\title{
Exercise training does not alter acetylcholine-induced responses in isolated pulmonary artery from rat
}

\author{
Y. Mitani, J. Maruyama, K. Maruyama, M. Sakurai
}

\begin{abstract}
Exercise training does not alter acetylcholine-induced responses in isolated pulmonary artery from rat. Y. Mitani, J. Maruyama, K. Maruyama, M. Sakurai. (C)ERS Journals Ltd 1999.

ABSTRACT: In chronic exercise-trained animals, acetylcholine (ACh)-stimulated endothelial nitric oxide (NO) release is enhanced in the systemic circulation. The purpose of the present study was to determine whether chronic exercise training also enhances NO-mediated relaxation in rat pulmonary artery.

Sprague-Dawley rats were randomly divided into groups of exercise-trained and sedentary control rats. The exercise-trained rats ran on a motor-driven treadmill at 30 $\mathrm{m} \cdot \mathrm{min}^{-1}$ up a $15^{\circ}$ incline $10-60 \mathrm{~min} \cdot \mathrm{day}^{-1}, 5$ days per week for 10 weeks, and had less body weight, lower serum total cholesterol and triglyceride levels than sedentary rats.

Contraction induced by potassium chloride and prostaglandin $(P G) F_{2 \alpha}$ were similar between isolated conduit pulmonary arterial rings from sedentary and exercisetrained rats. There were no differences between $\mathbf{P G F}_{2 \alpha}$-precontracted rings from sedentary and exercise trained rats in both $\mathrm{ACh}$ and sodium nitroprusside-induced relaxations. The NO synthase inhibitor, nitro-L-arginine, suppressed $\mathrm{ACh}$-induced relaxation in both sedentary and exercise-trained rats.

These results suggested chronic exercise training did not alter the acetylcholineinduced endothelial NO production and release and the sensitivity of vascular smooth muscle cell to NO in isolated conduit pulmonary artery of rat.

Eur Respir J 1999; 13: 622-625.
\end{abstract}

Exercise increases cardiac output and pulmonary blood flow, which elicit additional shear stress on the systemic and pulmonary vascular endothelial cells. In highly trained athletes, acetylcholine (ACh)-induced nitric oxide-mediated vasodilation in the forearm is enhanced compared to age-matched sedentary subjects [1]. In chronic exercisetrained animals, agonist-stimulated endothelial NO release is enhanced in the thoracic [2] and abdominal aorta [3] of rats, the thoracic aorta and pulmonary artery of New Zealand white rabbits [4], the epicardial coronary artery of dogs [5], and the coronary resistance arteries of pigs [6]. Several studies have shown that NO synthase gene expression increases in aortic endothelial cells from dogs after chronic exercise [7] and rats with chronic high flow via an arteriovenous fistula [8]. On the other hand, chronic exercise did not alter endothelium-dependent relaxation in the carotid artery of Wistar Kyoto rats [2] and the coronary artery of dogs [9]. Thus, the effect of exercise training on endothelial NO release might be different between the species or vascular bed examined.

In the thoracic aorta of spontaneously hypertensive rats, the endothelium-dependent response is impaired and chronic exercise increased endothelial NO release [2]. Thus, in the systemic circulation, exercise training might enhance endothelial NO release in structurally and functionally abnormal vascular beds as well as in the normal vascular beds. These observations partly suggest the benefit of chronic exercise in prevention of cardiovascular disorders
Depts of Pediatrics, Physiology, Anesthesiology, and the Intensive Care Unit, Mie University School of Medicine, Mie, Japan.

Correspondence: K. Maruyama, Dept of Anesthesiology, Mie University School of Medicine, 2-174, Edobashi, Tsu, 514 Mie, Japan, Fax: 01181592315227

\section{Keywords: Exercise}

hypertension

nitric oxide

pulmonary artery

Received: January 151998

Accepted after revision October 141998

Supported in part by Grants-in-aid for Scientific Research (05671259, 07771228 , 07457354, 09770038) from the Japanese Ministry of Education, Science and Culture; by a Grant for Pediatric Research (8C-02) from the Ministry of Health and Welfare; and by a Grant from the Mie Medical Research Foundation. such as hypertension and coronary artery disease. If exercise training enhances the endothelial release of NO in the pulmonary vasculature, it might be of benefit not only in the systemic and coronary circulation but also in the pulmonary circulation, since endothelium-dependent relaxation is impaired in hypertensive pulmonary vascular disease $[10,11]$. Since the rat is widely used as an experimental model of chronic pulmonary hypertension, it is interesting to study the effect of chronic exercise on rat pulmonary artery. Therefore, the ACh (endothelium-dependent relaxant) and sodium nitroprusside (SNP; endothelium-independent relaxant)-induced relaxation were investigated in isolated pulmonary arteries of rats after chronic exercise to determine whether chronic exercise alters the NO-mediated response in rat pulmonary artery.

\section{Materials and methods}

\section{Animals and training}

Male Sprague-Dawley rats (Clea, Japan) weighing 170$200 \mathrm{~g}$ (7-week-old) were used. Rats were randomly assigned to one of two groups after a 1-week familiarization period: exercise-trained (ET) rats $(n=9)$, and age-matched sedentary (SED) rats $(n=7)$. Rats were housed under a 12-h light-dark cycle and were fed standard rat food and water ad libitum. The exercise training was performed according to the protocol of DeLP et al. [3]. The ET rats ran on a 
Table 1. - Body weight (BW), total serum cholesterol (sChol) and triglyceride (sTrig), and heart weight (HW)

\begin{tabular}{|c|c|c|c|c|c|c|c|c|}
\hline & \multicolumn{4}{|c|}{ Body weight $g$} & \multirow{2}{*}{$\begin{array}{c}\mathrm{sChol} \\
\mathrm{mg} \cdot \mathrm{dL}^{-1}\end{array}$} & \multirow{2}{*}{$\begin{array}{c}\text { sTrig } \\
\mathrm{mg} \cdot \mathrm{dL}^{-1}\end{array}$} & \multirow{2}{*}{$\begin{array}{c}\mathrm{HW} / \mathrm{BW} \\
\mathrm{mg} \cdot \mathrm{g}^{-1}\end{array}$} & \multirow{2}{*}{$\mathrm{RV} / \mathrm{LV}+\mathrm{S}$} \\
\hline & Initial & Week 1 & Week 5 & Week 10 & & & & \\
\hline SED & $224 \pm 1$ & $\underset{0}{272 \pm 3}$ & $373 \pm 6$ & $433 \pm 4$ & $70 \pm 3$ & $215 \pm 28$ & $2.22 \pm 0.04$ & $0.24 \pm 0.01$ \\
\hline $\begin{array}{r}n \\
\mathrm{ET}\end{array}$ & $\begin{array}{c}9 \\
220 \pm 3\end{array}$ & $\begin{array}{c}9 \\
256 \pm 6^{*}\end{array}$ & $\begin{array}{c}9 \\
310 \pm 4^{+}\end{array}$ & $\begin{array}{c}9 \\
347 \pm 8^{+}\end{array}$ & $\begin{array}{c}8 \\
55 \pm 4 *\end{array}$ & $\begin{array}{c}8 \\
91 \pm 11^{+}\end{array}$ & $\begin{array}{c}6 \\
2.61 \pm 0.02^{+}\end{array}$ & $\begin{array}{c}7 \\
0.25 \pm 0.01\end{array}$ \\
\hline $\mathrm{n}$ & 7 & 7 & 7 & 7 & 7 & 7 & 7 & 6 \\
\hline
\end{tabular}

Values are mean \pm SEM; $n$ : number of rats; SED: sedentary group; ET: exercise-trained group; $\mathrm{RV} / \mathrm{LV}+\mathrm{S}$ : right ventricle/left ventricle plus septum weight ratio. ${ }^{*}: \mathrm{p}<0.05 .^{+}: \mathrm{p}<0.01$, compared to SED.

motor-driven treadmill (Columbus Instruments, OH, USA) at $30 \mathrm{~m} \cdot \mathrm{min}^{-1}$ up a $15^{\circ}$ incline 5 days per week. The length of time on the treadmill started at $10 \mathrm{~min} \cdot \mathrm{day}^{-1}$ for the first week, increasing to $20 \mathrm{~min} \cdot \mathrm{day}^{-1}$ for the second week, 30 $\mathrm{min} \cdot \mathrm{day}^{-1}$ for the third week, $40 \mathrm{~min} \cdot \mathrm{day}^{-1}$ for the fourth week, $50 \mathrm{~min} \cdot \mathrm{day}^{-1}$ for the fifth week, and progressed to a maximum of $60 \mathrm{~min} \cdot$ day $^{-1}$ by the sixth week. The length of time on the treadmill was then maintained at $60 \mathrm{~min} \cdot$ day $^{-1}$ for an additional 4 weeks. Thus, the ET rats underwent exercise training for 10 weeks in total. All rats were weighed at the beginning of training and once a week until they were sacrificed for the isolation of the pulmonary arteries. Institutional approval of animal investigation was obtained. Table 1 lists the mean body weight (BW) for each group.

\section{Preparation of pulmonary arteries}

Rats were anaesthetized with intraperitoneal pentobarbital $\left(50 \mathrm{mg} \cdot \mathrm{kg}^{-1}\right)$. The lungs and heart were removed en bloc and placed in modified Krebs-Henseleit solution at room temperature. The composition of this solution was $\mathrm{NaCl} 115.0 \mathrm{mM}$; KCl $4.7 \mathrm{mM} ; \mathrm{CaCl}_{2} 2.5 \mathrm{mM} ; \mathrm{MgCl}_{2} 1.2$ $\mathrm{mM} ; \mathrm{NaHCO}_{3} 25.0 \mathrm{mM} ; \mathrm{KH}_{2} \mathrm{PO}_{4} 1.2 \mathrm{mM}$; and dextrose $10.0 \mathrm{mM}$. The right ventricle (RV) of the heart was dissected from the left ventricle plus septum $(\mathrm{LV}+\mathrm{S})$ and these cardiac portions were weighed separately. The values for $\mathrm{RV} /(\mathrm{LV}+\mathrm{S})$ and heart weight/body weight were then calculated to determine whether ventricular hypertrophy had developed. Two pulmonary artery segments, a left extrapulmonary artery (EPA, 1.4-1.6 mm external diameter) and an intrapulmonary artery (IPA, $0.7-1.1 \mathrm{~mm}$ external diameter), were isolated and gently freed from fat and connective tissue. Ring segments $(2 \mathrm{~mm})$ were cut (1-2 rings from the EPA and 2-4 rings from the IPA) and suspended vertically between hooks in organ baths $(20 \mathrm{~mL})$ containing modified Krebs-Henseleit solution; the solution was maintained at $37^{\circ} \mathrm{C}$ and bubbled with a mixture of $95 \%$ air and $5 \% \mathrm{CO}_{2}$.

In preliminary experiments, active and resting tension relationships were obtained by increasing the resting tension, using a range of forces between 0.25 and $2.5 \mathrm{~g}$, according to the method of ToDA et al. [12]. The optimal resting tension for vasodilation studies was $0.75 \mathrm{~g}$ for EPA and IPA rings from both ET and SED rats. At this resting tension, the peak contraction was obtained in response to $70 \mathrm{mM} \mathrm{KCl}$. In all experiments, changes in isometric force were measured with a force displacement transducer (TB 612; Nihon Kohden, Tokyo, Japan) connected to a carrier amplifier (AP600G; Nihon Kohden) and were recorded on a pen recorder (MC 6622; Watanabe, Tokyo, Japan).

\section{Vasodilation studies}

Pulmonary artery rings under the optimal resting tension were washed every $15-20 \mathrm{~min}$ and allowed to equilibrate for $2 \mathrm{~h}$. After the equilibration period, $70 \mathrm{mM} \mathrm{KCl}$ contraction curves were routinely recorded twice as a measure of maximal contractility with the contraction shown by the second curve used as the maximal contraction. A cumulative concentration-response curve to prostaglandin $(\mathrm{PG}) \mathrm{F}_{2 \alpha},\left(10^{-8}-10^{-5} \mathrm{M}\right)$ was obtained, and the approximate concentration of $\mathrm{PGF}_{2 \alpha}$ needed to produce $50 \%$ of the maximal contraction induced by $70 \mathrm{mM} \mathrm{KCl}$ (EC50) was determined. The rings were then washed every $15 \mathrm{~min}$, equilibrated for $1 \mathrm{~h}$ in total, and precontracted with $\mathrm{PGF}_{2 \alpha}$ $\left(10^{-6}-10^{-5} \mathrm{M}\right)$ to obtain $50-70 \%$ of the maximal contraction induced by $70 \mathrm{mM} \mathrm{KCl}$. Following $\mathrm{PGF}_{2 \alpha}$-induced precontraction with or without a NO synthase inhibitor, nitro-L-arginine (L-NA, $10^{-4} \mathrm{M}$ ), a cumulative concentration-response curve was obtained for $\mathrm{ACh}\left(10^{-8}\right.$ $10^{-5} \mathrm{M}$ ) by producing a stepwise increase in the ACh concentration as soon as a stable response to each preceding level was reached. Finally, $10^{-4} \mathrm{M}$ papaverine was added to produce maximal relaxation. Then the rings without L-NA were washed every $15 \mathrm{~min}$ and equilibrated for a total of 1 $\mathrm{h}$ and precontracted again with $\mathrm{PGF}_{2 \alpha}$, and a cumulative concentration-response curve was obtained for a second vasodilator, SNP $\left(10^{-9}-10^{-5} \mathrm{M}\right)$. Finally, $10^{-4} \mathrm{M}$ papaverine was again added to produce maximal relaxation. The results were expressed as percentage relaxation of each papaverine-induced maximal relaxation and these percentages were plotted against the negative logarithmic values of the drug doses. The response to ACh was examined in the presence of $10^{-6} \mathrm{M}$ indomethacin to eliminate vasodilation due to prostacyclin release.

\section{Reagents}

The following drugs were used: ACh hydrochloride, (Wako Pure Chemical Industries, Osaka, Japan); SNP, indomethacin, and L-NA (Sigma); and PGF $_{2 \alpha}$ (Ono Pharmaceutical Co., Osaka, Japan). The concentrations of the drugs were expressed as the final molar concentrations in the organ bath.

\section{Data analysis}

Results were expressed as mean \pm SEM. Numbers in parentheses (n) are the number of rings from seven SED or six ET rats. Differences between SED and ET rats were determined by the unpaired Student's t-test. When more than two means were compared, one-way analysis of variance was used. If a significant difference was found, Scheffe's 

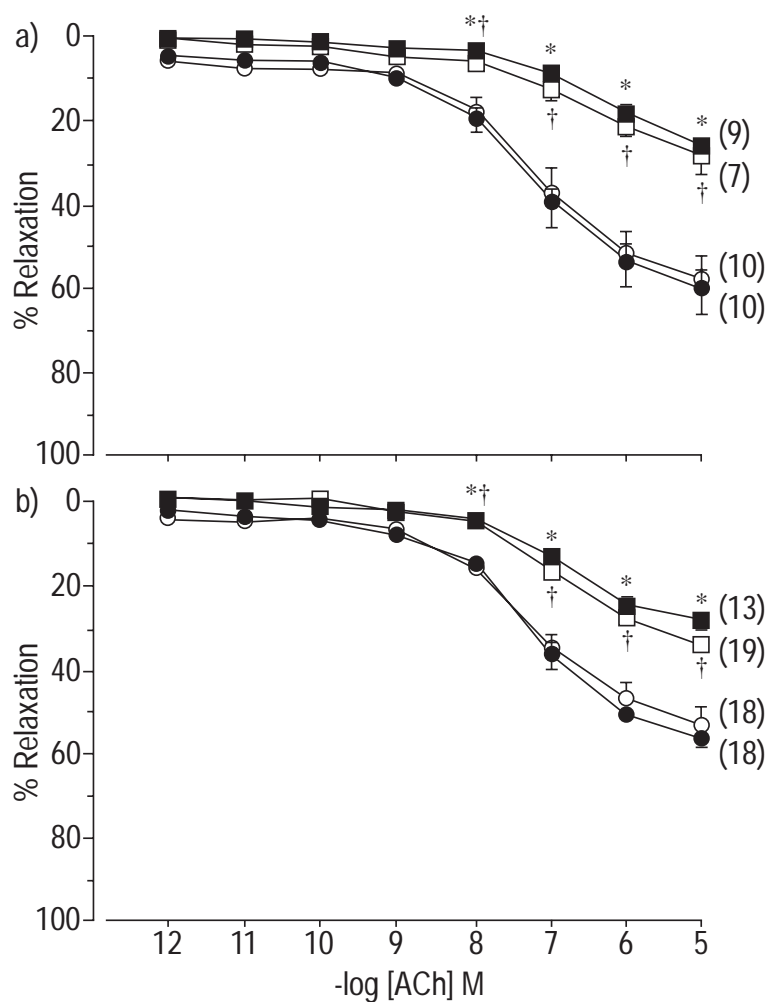

Fig. 1. - Relaxation in response to acetylcholine (ACh) in precontracted rings of a) extrapulmonary artery and b) intrapulmonary artery from sedentary (SED) rats with $(\square)$ and without (O) L-NA and exercisetrained (ET) rats with $(\boldsymbol{\square})$ and without $(\bullet)$ L-NA. The rings were precontracted with prostaglandin $\mathrm{F}_{2 \alpha}$ to obtain $50-70 \%$ of the maximal contraction induced by $70 \mathrm{mM} \mathrm{KCl}$. Relaxation induced by $10^{-4} \mathrm{M}$ papaverine was taken as $100 \%$. There were no differences between groups. L-NA $\left(10^{-4} \mathrm{M}\right)$, suppressed ACh-induced relaxation in both SED and $\mathrm{ET}$ rats. Values were mean \pm SEM; numbers in parentheses are number of rings from seven SED or six ET rats. *: $\mathrm{p}<0.05$ versus ET rats without $\mathrm{L}-\mathrm{NA} ;{ }^{\dagger}: \mathrm{p}<0.05$ versus SED rats without $\mathrm{L}-\mathrm{NA}$.

F-test was used to identify which groups were different. A p-value $<0.05$ was considered to indicate statistical significance.

\section{Results}

Body weight, right ventricular hypertrophy and serum total cholesterol and triglyceride levels

SED rats gained weight throughout the experiment. The ET rats gained less body weight than SED rats at each time point during the exercise training (table 1). The heart weight/body weight ratio was greater in ET rats probably owing to lower body weight in ET rats. There was no difference in the $\mathrm{RV} / \mathrm{LV}+\mathrm{S}$ ratio between ET rats and SED rats (table 1). Serum total cholesterol (sChol) and triglyceride (sTrig) levels were significantly lower in ET rats than in SED rats (table 1).

Response to $70 \mathrm{mM}$ potassium chloride and prostaglandin $F_{2 \alpha}$

Mean absolute values of contraction induced by $70 \mathrm{mM}$ $\mathrm{KCl}$ were similar between rings from SED rats and ET rats: $387 \pm 13(\mathrm{n}=12)$ versus $387 \pm 13(\mathrm{n}=12) \mathrm{mg}$ in EPA; $367 \pm 12$ $(\mathrm{n}=21)$ versus $394 \pm 30(\mathrm{n}=16) \mathrm{mg}$ in IPA. $\mathrm{PGF}_{2 \alpha}$ induced dose-dependent contractions in EPA and IPA rings, which were similar between SED rats and ET rats. Concentrations of $\mathrm{PGF}_{2 \alpha}\left(-\log \left[\mathrm{PGF}_{2 \alpha}\right]\right)$ needed to produce EC50 were similar between SED rats and ET rats: $5.8 \pm 0.1(\mathrm{n}=12)$ versus $5.8 \pm 0.1(\mathrm{n}=12)$ in EPA; $5.7 \pm 0.0(\mathrm{n}=21)$ versus $5.7 \pm$ $0.1(\mathrm{n}=16)$ in IPA.

\section{Relaxation by acetylcholine and sodium nitroprusside}

In both EPA and IPA rings from SED rats, ACh (fig. 1) and SNP (fig. 2) induced relaxation in a concentrationdependent manner. There were no differences in relaxation between rings from SED and ET rats in both ACh and SNP-induced relaxation. ED50 values for ACh without L-NA (-log[Ach]) and SNP (-log[SNP]) (concentration of Ach to produce $50 \%$ of maximal relaxation of Ach and SNP) were similar between SED and ET rats. ACh (EPA, 7.4 $\pm 0.2(\mathrm{n}=10)$ in SED rats versus $7.6 \pm 0.2(\mathrm{n}=10)$ in ET rats; IPA, $7.4 \pm 0.1(\mathrm{n}=18)$ in SED rats versus $7.4 \pm$ $0.1(\mathrm{n}=18)$ in ET rats); SNP (EPA, $7.5 \pm 0.2(\mathrm{n}=8)$ in SED rats versus $7.6 \pm 0.1(\mathrm{n}=10)$ in ET rats; IPA, $7.9 \pm 0.5(\mathrm{n}=20)$ in SED rats versus $7.5 \pm 0.2(\mathrm{n}=12)$ in ET rats). L-NA $\left(10^{-4}\right.$ M) markedly inhibited ACh-induced relaxation in both SED and ET rats (fig. 1). ED50 of ACh with L-NA was higher than without L-NA $(\mathrm{p}<0.05)(\mathrm{EPA}, 7.0 \pm 0.3 \quad(\mathrm{n}=7)$ in SED rats, 6.5 $\pm 0.2(\mathrm{n}=9)$ in ET rats; IPA, 6.9 $\pm 0.1(\mathrm{n}=$ $19)$ in SED rats, $7.0 \pm 0.1(n=13)$ in ET rats $)$.

\section{Discussion}

ACh-induced relaxation was not altered by chronic exercise. Relaxation to SNP was not altered in isolated conduit pulmonary artery from ET rats, suggesting that sensitivity of guanylate cyclase to NO was not altered in the pulmonary vascular smooth muscle of ET rats and that relaxation due to $\mathrm{ACh}$ in this vessel could be the measure of the pulmonary endothelial NO production and/or release in ET rats. Thus, exercise training did not alter ACh-induced endothelial NO production and/or its release in isolated rat pulmonary artery. Since L-NA suppressed, but did not eliminate, the relaxation effects of $\mathrm{ACh}$, it is probable that a vasodilatory substance other than NO might also be produced in response to ACh.

In ET rats, endothelium-dependent relaxation is enhanced in conduit (thoracic and abdominal aorta) arteries $[2,3]$, but not in the conduit pulmonary artery in the present study. The aorta is exposed to higher pulse pressure and/or mean flow velocity compared to the pulmonary artery [13], raising the possibility that aortic endothelial cells respond differently to chronic exercise than pulmonary endothelial cells. Although the values of cardiac output and pulmonary blood flow are the same, the degree of shearstress may be different between the aorta and pulmonary artery. Shear-stress is determined by blood flow velocity and blood vessel diameter [8]. Chronic high flow produced by an arteriovenous fistula does not enhance NO release from the pulmonary arterial endothelial cells of dogs [14], which is similar to the present study. However, it is not possible to demonstrate that NO release in response to agonists other than $\mathrm{ACh}$ is unaltered after chronic exercise. The chronic exercise-induced alteration of endothelial function in the smaller micropulmonary vasculature remains to be determined. In ET rabbits, ACh-induced relaxation in isolated pulmonary artery was enhanced slightly but significantly compared to control SED rabbits [4], disagreeing 


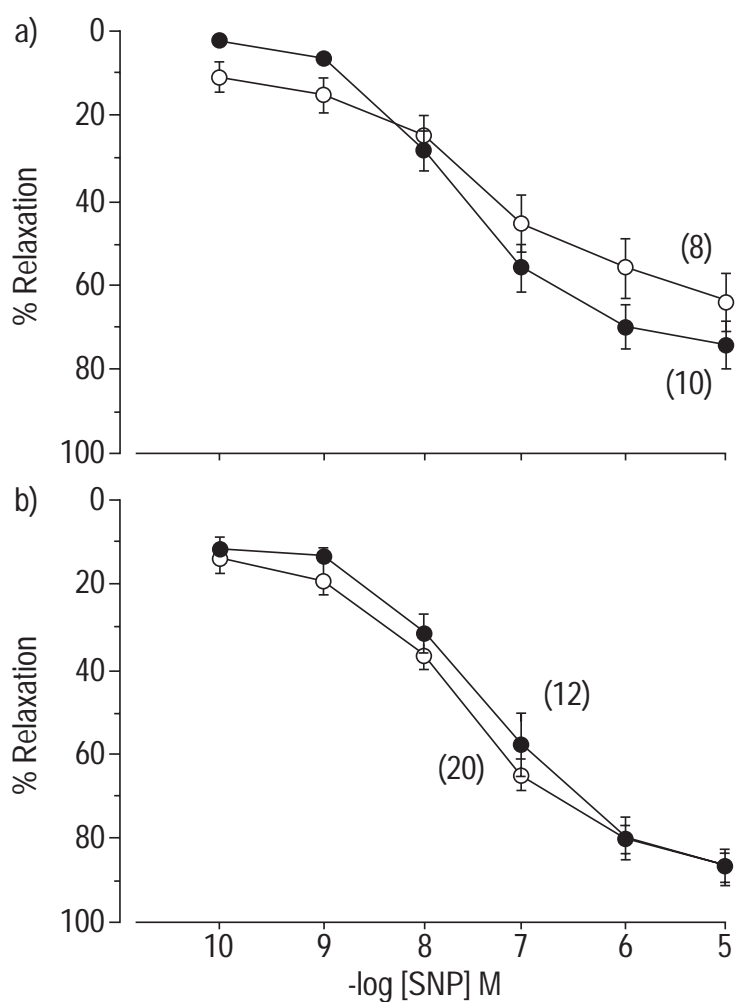

Fig. 2. - Relaxation response to sodium nitroprusside (SNP) in precontracted rings of a) extrapulmonary artery and b) intrapulmonary artery from sedentary rats $(\bigcirc)$ and exercise trained rats $(\bullet)$. The rings were pre-contracted with prostaglandin $\mathrm{F}_{2 \alpha}$ to obtain $50-70 \%$ of the maximal contraction induced by $70 \mathrm{mM} \mathrm{KCl}$. Relaxation induced by $10^{-4}$ $\mathrm{M}$ papaverine was taken as $100 \%$. There were no differences between groups. Values were mean \pm SEM; numbers in parentheses are number of rings from seven SED or six ET rats.

with the present results in rats. There might be species differences to account for this.

ET induced lower sChol and sTrig levels and higher heart weight/body weight ratios than in SED rats, consistent with earlier studies $[1,15,16]$. These results partly showed the efficacy of the training protocol in the present study. As the study progressed, the ET rats showed a much lower body weight gain compared to SED rats. It is notable that the exercise routine caused a marked stunting in normal growth, suggesting that energy demands have been excessive and that rats had been undergoing a degree of metabolic starvation. Metabolic starvation might affect free radical production. In the present study, SED rats without food restriction might not have been a true control. Possibly owing to this concern, food management is different among studies dealing with exercise. In the study by DeLP et al. [3], ET rats continued to be fed ad libitum during the training period while food was restricted for SED rats to ensure matched body weight between the two groups. In other studies both ET and SED rats (4-7 week-old rats) were fed ad libitum, resulting in a lower body weight in ET rats $[2,16,17]$. The results were similar in the studies comparing exercise training enhanced endothelium-dependent relaxation of aortic rings from ET rats and SED rats with and without food restriction.

In summary, chronic exercise training did not alter the acetylcholine (endothelium-dependent relaxant) and so- dium nitroprusside (endothelium-independent relaxant)induced pharmacological relaxation, at least in conduit pulmonary arteries, in rats. The effect of exercise training on smaller pulmonary vasculature and/or shear stress-induced functional relaxation in the in vivo situation remains to be determined.

\section{References}

1. Kingwell BA, Tran B, Cameron JD, Jennings GL, Dart AM. Enhanced vasodilation to Ach in athletes is associated with lower plasma cholesterol. Am J Physiol 1996; 270: H2008-H2013.

2. Chen H-I, Chiang I-P. Chronic exercise decreases adrenergic agonist-induced vasoconstriction in spontaneously hypertensive rats. Am J Physiol 1996; 271: H977-H983.

3. Delp MD, McAllister RM, Laughlin MH. Exercise training alters endothelium-dependent vasoreactivity of rat abdominal aorta. J Appl Physiol 1993; 75: 1351-1363.

4. Chen H-I, Li H-T. Physical conditioning can modulate endothelium-dependent vasorelaxation in rabbits. Arterioscler Thromb 1993; 13: 852-856.

5. Wang J, Wolin MS, Hintze TH. Chronic exercise enhances endothelium-mediated dilation of epicardial coronary artery in conscious dogs. Circ Res 1993; 73: 829-838.

6. Muller JM, Myers PR, Laughlin H. Vasodilator responses of coronary resistance arteries of exercise-trained pigs. Circulation 1994; 89: 2308-2314.

7. Sessa WCK, Pritchard N, Seyedi N, Wang J, Hintze TH. Chronic exercise in dogs increases coronary vascular nitric oxide production and endothelial cell nitric oxide synthase gene expression. Circ Res 1994; 74: 349-353.

8. Nadaud S, Philippe M, Arnal J-F, Michel J-B, Soubrier F. Sustained increase in aortic endothelial nitric oxide synthase expression in vivo in a model of chronic high blood flow. Circ Res 1996; 79: 857-863.

9. Rogers PJ, Miller TD, Bauer BA, Brum JM, Bove AA, Vanhoutt PM. Exercise training and responsiveness of isolated coronary arteries. J Appl Physiol 1991; 71: 23462350.

10. Dinh-Xuan AT, Higenbottam TW, Clelland CA, et al. Impairment of endothelium-dependent pulmonary artery relaxation in chronic obstructive lung disease. $N$ Engl J Med 1991; 324: 1539-1547.

11. Maruyama J, Maruyama K. Impaired nitric oxide-dependent responses and their recovery in hypertensive pulmonary arteries of rats. Am J Physiol 1994; 266 : H2476-H2488.

12. Toda N, Hayashi S. Age-dependent alteration in response of isolated rabbit basilar arteries to vasoactive agents. $J$ Pharmacol Exp Ther 1979; 211: 716-721.

13. Tschudi MR, Barton M, Bersinger NA, et al. Effect of age on kinetics of nitric oxide release in rat aorta and pulmonary artery. J Clin Invest 1996; 98: 899-905.

14. Fullerton DA, Mitchell MB, Jones DN, Maki A, McIntyre RC. Pulmonary vasomotor dysfunction is produced with chronically high pulmonary blood flow. J Thorac Cardiovasc Surg 1996; 111: 190-197.

15. Lopez-SA, Vial R, Balart L, Arroyave G. Effect of exercise and physical fitness on serum lipids and lipoproteins. Atherosclerosis 1974; 20: 1-9.

16. Koller A, Huang A, Sun D, Kaley G. Exercise training augments flow-dependent dilation in rat skeletal muscle arterioles: role of endothelial nitric oxide and prostaglandins. Circ Res 1995; 76: 544-550.

17. Sun D, Huang A, Kollar A, Kaley G. Short-term daily exercise activity enhances endothelial NO synthesis in skeletal muscle arterioles of rats. J Appl Physiol 1994; 76 : 2241-2247. 